\title{
Heavy Hyperon-Antihyperon Production
}

\author{
W. Oelert*, D. Grzonka*, L. Jarczyk ${ }^{\dagger}$, K. Kilian*, P. Moskal ${ }^{\dagger}$ and P. Winter* \\ *IKP - Research Centre Jülich, D-52425 Jülich, Germany \\ ${ }^{\dagger}$ Institute of Physics, Jagellonian University, PL-30-059 Cracow, Poland
}

\begin{abstract}
Based on the experience from the production of light antihyperon-hyperon $(\bar{\Lambda} \Lambda, \bar{\Sigma} \Sigma)$ pairs at LEAR (experiment PS185) it is suggested to continue the investigations towards the heavier antihyperon-hyperon pairs $\bar{\Xi} \Xi$ and $\bar{\Omega} \Omega$ in view of:

1. the production dynamics of the heavier antihyperon-hyperon out of the $\bar{p} p$ annihilation

2. a comparison of the "( $3 s)(3 \bar{s})$ "- quark system $\bar{\Omega} \Omega$

to the $3(\bar{s} s)=3 \phi$ meson production,

where both systems the $\bar{\Omega} \Omega$ and the $3 \phi$ have similar masses

(3.345 and 3.057, respectively) and identical valence quark content.
\end{abstract}

\begin{abstract}
A systematic study of the antihyperon-hyperon production with increasing strangeness content is interesting for the following reasons: The $\bar{\Omega} \Omega$ production is the creation of two spin $3 / 2$ objects out of the two spin $1 / 2 \bar{p} p$ particles. Results of the PS185 experiments prove a clear dominance of the spin triplet $\bar{s} s$ dissociation. In the $\Omega(\bar{\Omega})$ the three $s$-quarks (three $\bar{s}$-quarks) are aligned to spin $3 / 2$ each. If the three $\bar{s}$ pairs are now all in spin triplet configurations when created out of the gluonic interaction they should have spin parity quantum number as $3^{-}$as long as $\Omega \bar{\Omega}$ is created with relative $L=0$ angular momentum.

The comparison of the $\Omega \bar{\Omega}$ baryon pair to the $\phi \phi \phi$ three meson production (where the three $\bar{s} s$ quark pairs might not but can be produced without relative correlation) would provide a unique determination of the intermediate matter state.

Measurements of excitation functions and polarization transfers should be used to examine these gluon rich $\bar{p} p \rightarrow \bar{\Omega} \Omega$ and $\bar{p} p \rightarrow \phi \phi \phi$ reaction channels.

Such experiments should be performed at the PANDA detector at the FAIR facility of the GSI.
\end{abstract}

\section{INTRODUCTION}

Interest for studying the production of hyperon-antihyperon pairs following antiprotonproton annihilations is based largely on the aim to understand the nature of flavour production and its dynamics. There is a large amount of data from the PS185 [1] collaboration as reported during the LEAP-05 conference by T. Johansson [2] and J.M. Richard [3]. The goal of those PS185 experiments is to establish the definitive $\bar{p} p \rightarrow \bar{Y} Y$ data set in the low-energy regime. From the theoretical analysis of the data, one hopes to gain insight into the behaviour of hadron interactions at intermediate energies, i.e. in an energy regime where perturbative QCD is inappropriate and both quark-gluon and meson degrees of freedom are believed to be important.

Heavy hyperon-antihyperon production studies are relevant since i) rather little is known about heavy flavour hyperons, even down to the final proof of their existence; ii) different hyperon-antihyperon pairs are produced in distinct but specific hadronic environments; iii) threshold production studies keep the interpretation as simple as 
possible and iv) quark dynamic effects can be observed. The special interest for the investigation of $\bar{\Omega} \Omega$ production will be discussed in this contribution.

\section{RESULTS FROM THE PS185 EXPERIMENT}

Results of the PS185-collaboration studies - as far as relevant for the recent discussion are shown in figure 1 (left) and (right), demonstrating that the differential cross sections for the hyperon-antihyperon pair production following the $\bar{p} p$ annihilation feature the onset of higher partial waves already at very low excess energies and that the singlet fraction for the $\bar{p} p \rightarrow \bar{\Lambda} \Lambda$ reaction is largely consistent with zero, respectively.
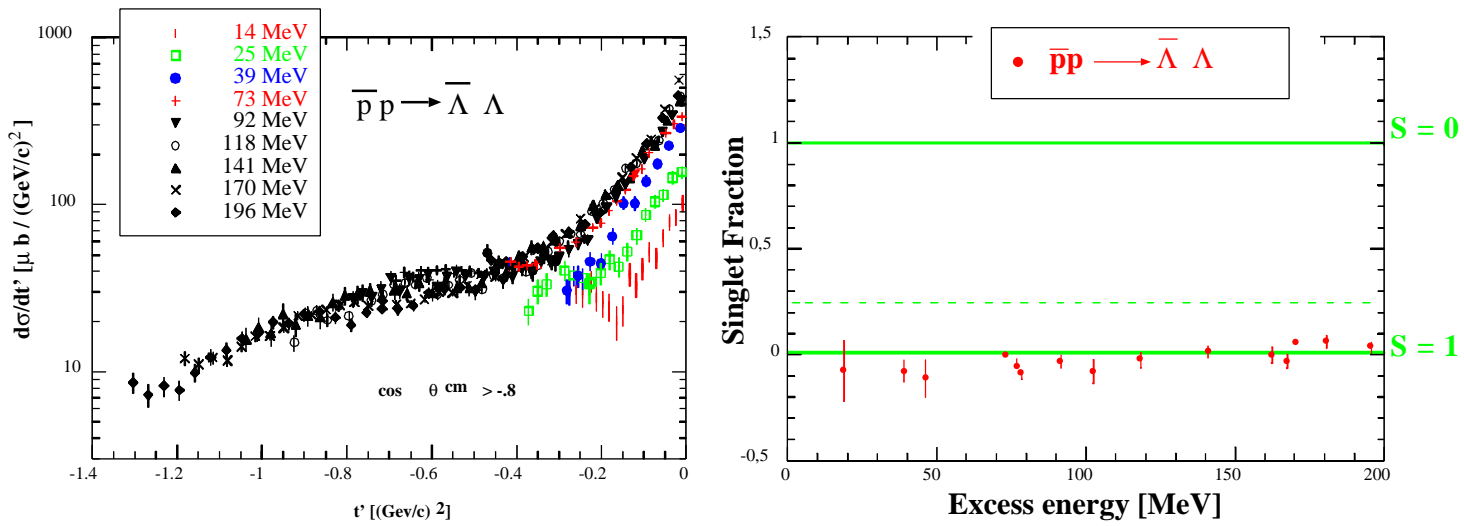

FIGURE 1. Left: PS185 results of differential cross sections at various energies, shown as a function of momentum transfer. Right: PS185 results of the singlet fractions for the reaction: $\bar{p} p \rightarrow \bar{\Lambda} \Lambda$ at various energies, shown as a function of the excess energy

\section{FROM LIGHT TO HEAVY HYPERONS}

Based on the experimental and theoretical experience from the production of light antihyperon-hyperon pairs at LEAR it is suggested to measure the production of both heavy antihyperon-hyperon pairs and the three $\phi$-meson system at the future facility FAIR at GSI. The foreseen momentum range [4] of the $\bar{p}$-beam at the HESR/FAIR allows to study hyperons with one, two and three valence s-quarks or one valence cquark up to the $\Xi_{c}$ with its mass of $\mathrm{M}=2.47 \mathrm{GeV} / \mathrm{c}^{2}$ in the $\bar{p} p$ interaction, see table 1 .

The common special feature of the hyperons is their weak (flavour changing) decay with $c \tau$ values of a few centimeters, as presented in table 2. Typical decay schemes of the lighter hyperons might be represented in two categories. The charged hyperons (antihyperons) decay mainly (see figure 2) delayed into a charged meson and a $\Lambda(\bar{\Lambda})$ which then decays to the two charged particles $\pi^{-}$and $p\left(\pi^{+}\right.$and $\left.\bar{p}\right)$. The neutral hyperons (antihyperons) decay via $\Lambda+\pi^{0}\left(\bar{\Lambda}+\pi^{0}\right)$ into the two charged particle system 
TABLE 1. Relevant parameters for the production of hyperons in $\bar{p} p$ annihilation

\begin{tabular}{|c|c|c|c|}
\hline Hyperon & $\begin{array}{r}\text { Mass } \\
\left(\mathrm{MeV} / \mathrm{c}^{2}\right)\end{array}$ & $\begin{array}{r}\sqrt{s} \\
(\mathrm{MeV})\end{array}$ & $\begin{array}{r}\text { Beam } \\
\text { Momentum } \\
(\mathrm{GeV} / \mathrm{c})\end{array}$ \\
\hline$\Lambda$ & $1115.57 \pm 0.06$ & 2231.14 & 1.435 \\
\hline$\Sigma^{+}$ & $1189.37 \pm 0.06$ & 2378.74 & 1.854 \\
\hline$\Sigma^{0}$ & $1192.55 \pm 0.10$ & 2385.1 & 1.871 \\
\hline$\Sigma^{-}$ & $1197.50 \pm 0.05$ & 2395.0 & 1.900 \\
\hline$\Xi^{0}$ & $1314.80 \pm 0.8$ & 2629.60 & 2.582 \\
\hline$\Xi^{-}$ & $1321.34 \pm 0.14$ & 2642.68 & 2.621 \\
\hline$\Omega^{-}$ & $1672.43 \pm 0.14$ & 3344.86 & 4.936 \\
\hline$\Lambda_{c}^{+}$ & $2285.2 \pm 1.2$ & 4570.4 & 10.150 \\
\hline$\Sigma_{c}^{0}$ & $2452.7 \pm 1.3$ & 4905.4 & 11.848 \\
\hline$\Sigma_{c}^{++}$ & $2453.0 \pm 1.2$ & 4906.0 & 11.851 \\
\hline$\Sigma_{c}^{+}$ & $2453.2 \pm 1.2$ & 4906.4 & 11.853 \\
\hline$\Xi_{c}^{+}$ & $2466.5 \pm 2.5$ & 4933.0 & 11.993 \\
\hline$\Xi_{c}^{0}$ & $2473.1 \pm 2.0$ & 4945.2 & 12.063 \\
\hline$\Omega_{c}^{0}$ & $2740 . \pm 2.0$ & 5480. & 15.1 \\
\hline
\end{tabular}

$\pi^{-}$and $p\left(\pi^{+}\right.$and $\left.\bar{p}\right)$ and $\gamma$-quanta. The rather simple decay features allow an effective neutral or charge-multiplicity-step trigger. It is understood, however, that the hyperons with quarks heavier than the strange quark do not have a unique decay scheme any more and the restriction to particular decay channels seems to be appropriate.

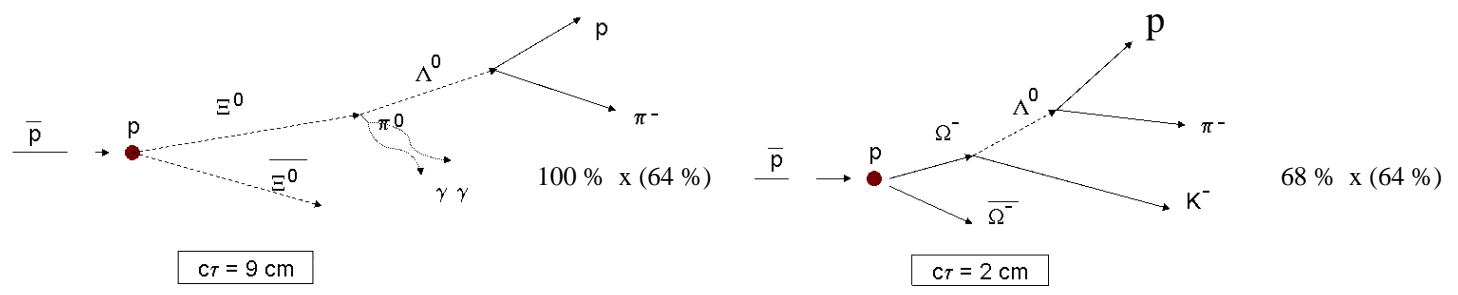

FIGURE 2. Decay scheme of the two hyperons $\Xi^{0}$ and $\Omega^{-}$

\section{THE SYSTEM: $3 \times S$ AND $3 \times \bar{S}$ QUARKS}

A systematic study of antihyperon-hyperon production with increasing strangeness (charm) content is certainly interesting due to the change of the quark dynamics in the different flavour environment of hadronic matter.

Besides the general interest of this research the production of the six-quark system with three $s$-quarks and three $\bar{s}$-quarks in the final state (i.e. the observation of the $\bar{\Omega} \Omega$ baryon pair and the $\phi \phi \phi$ three meson system) should be stressed. The angular distribution of $\bar{\Omega}$ (or $\Omega$ ) would be symmetric to $90^{\circ} \mathrm{c} . \mathrm{m}$. if the intermediate state is a compound like system state of gluonic matter with definite quantum numbers. If, 
TABLE 2. Some properties of hyperons [5]

\begin{tabular}{|c|c|c|c|c|c|c|c|}
\hline Hyperon & $\begin{array}{l}\text { Quark- } \\
\text { content }\end{array}$ & $\mathbf{I}$ & $\mathbf{J}^{\pi}$ & $\begin{array}{c}\text { Mass } \\
\left(\mathrm{MeV} / \mathrm{c}^{2}\right)\end{array}$ & $\begin{array}{l}\text { Mean life } \\
\left(\mathbf{s} \times 10^{-13}\right)\end{array}$ & $\begin{array}{c}c \tau \\
(\mathbf{c m})\end{array}$ & $\alpha_{\text {Main }}$ \\
\hline$\Lambda$ & uds & 0 & $\frac{1}{2}^{+}$ & $1115.57 \pm 0.06$ & $2632 \pm 20$ & 7.89 & $+0.642 \pm 0.013$ \\
\hline$\Sigma^{+}$ & uus & 1 & $\frac{1}{2}+$ & $1189.37 \pm 0.06$ & $799 \pm 4$ & 2.40 & $-0.980 \pm 0.015$ \\
\hline$\Sigma^{0}$ & uds & 1 & $\frac{1}{2}+$ & $1192.55 \pm 0.10$ & $7.4 \times 10^{-7}$ & & \\
\hline$\Sigma^{-}$ & dds & 1 & $\frac{1}{2}+$ & $1197.50 \pm 0.05$ & $1479 \pm 11$ & 4.40 & $-0.068 \pm 0.008$ \\
\hline$\Xi^{0}$ & uss & $\frac{1}{2}$ & $\frac{1}{2}+$ & $1314.80 \pm 0.8$ & $2900 \pm 90$ & 8.69 & $-0.411 \pm 0.022$ \\
\hline$\Xi^{-}$ & dss & $\frac{1}{2}$ & $\frac{1}{2}+$ & $1321.34 \pm 0.14$ & $1639 \pm 15$ & 4.91 & $-0.456 \pm 0.014$ \\
\hline$\Omega^{-}$ & sss & 0 & $\frac{\frac{2}{3}}{2}+$ & $1672.43 \pm 0.14$ & $822 \pm 12$ & 2.46 & $-0.026 \pm 0.026$ \\
\hline$\Lambda_{c}^{+}$ & udc & 0 & $\frac{1}{2}^{+}$ & $2285.2 \pm 1.2$ & $1.91 \pm 0.15$ & 0.006 & \\
\hline$\Sigma_{c}^{0}$ & ddc & 1 & $\frac{1}{2}+$ & $2452.7 \pm 1.3$ & & & \\
\hline$\Sigma_{c}^{++}$ & uuc & 1 & $\frac{1}{2}+$ & $2453.0 \pm 1.2$ & & & \\
\hline$\Sigma_{c}^{+}$ & udc & 1 & $\frac{1}{2}+$ & $2453.2 \pm 1.2$ & & & \\
\hline$\Xi_{c}^{+}$ & usc & $\frac{1}{2}$ & $\frac{1}{2}+{ }^{2}(?)$ & $2466.5 \pm 2.5$ & $3.0 \pm 1.0$ & 0.009 & \\
\hline$\Xi_{c}^{0}$ & $\mathrm{dsc}$ & $\frac{2}{2}$ & $\frac{1}{2}+(?)$ & $2473.1 \pm 2.0$ & $0.82 \pm 0.6$ & 0.002 & \\
\hline$\Omega_{c}^{0}$ & $\mathrm{ssc}$ & $\frac{2}{2}$ & $\frac{1}{2}+(?)$ & $2740 . \pm 2.0$ & & & \\
\hline
\end{tabular}

however, other production mechanisms as boson exchange probabilities are relevant, the symmetry via a pure intermediate gluonic matter state will be broken. It would be an interesting and important result to see whether there is a correlation between the initial $\bar{p}(p)$ and the final $\bar{\Omega} \Omega$.

Since the asymmetry parameter $\alpha$ of the $\Omega$ decay is very small (see table 2), its polarization features cannot be measured via the weak decay directly. The determination of the polarization of the weakly decaying daughter hyperons allows to extract spin observables for the $\Omega$ particle.

A distinct feature of the $\bar{\Omega} \Omega$ production is the creation of two spin $3 / 2$ objects out of the $\bar{p} p$ interaction. Results from the PS185 experiment proove a clear dominance of the triplet $\bar{s} s$ production at threshold. Since in the $\bar{\Omega}(\Omega)$ the three $s$-quarks ( $\bar{s}$-quarks) are oriented parallel, the three $\bar{s} s$ pairs created out of the gluonic intermediate state should have spin quantum number as $3^{-}$if the $\bar{\Omega}(\Omega)$ is created with relative $L=0$ angular momentum.

The comparison of the $\bar{\Omega}(\Omega)$ baryon pair to the $\phi \phi \phi$ three meson production (where the three $I^{G}\left(J^{P C}\right)=0^{-}\left(1^{--}\right) \bar{s} s$ mesons may not but can be produced with no relative correlation) would give valuable information for a unique determination of the intermediate matter state.

\section{REFERENCES}

1. B. Bassalleck et al., Phys. Rev. Lett. 89 (2002) 212302 and references therein.

2. T. Johansson, see contribution to the LEAP-05 conference in these proceedings.

3. J.M. Richard, see contribution to the LEAP-05 conference in these proceedings.

4. C. Schwarz et al., Physica Scripta T104 (2003) 147, see also http://www.gsi.de/fair/index.html 
5. S. Eidelman et al., Phys. Lett. B 592 (2004) 1. 